\title{
A novel approach for obtaining new identities for the $\lambda$ extension of q-Euler polynomials arising from the q-umbral calculus
}

\author{
Serkan Araci $^{\mathrm{a}, *}$, Mehmet Acikgoz ${ }^{\mathrm{b}}$, Toka Diagana ${ }^{\mathrm{c}}$, H. M. Srivastava ${ }^{\mathrm{d}, \mathrm{e}}$ \\ ${ }^{a}$ Department of Economics, Faculty of Economics, Administrative and Social Science, Hasan Kalyoncu University, TR-27410 Gaziantep, \\ Turkey. \\ ${ }^{b}$ Department of Mathematics, Faculty of Science and Arts, University of Gaziantep, TR-27310 Gaziantep, Turkey. \\ ${ }^{c}$ Department of Mathematics, Howard University, 2441 6th Street, NW Washington 20059, D.C., U.S.A. \\ ${ }^{d}$ Department of Mathematics and Statistics, University of Victoria, Victoria, British Columbia V8W 3R4, Canada. \\ ${ }^{e}$ China Medical University, Taichung 40402, Taiwan, Republic of China.
}

Communicated by Sh. Wu

\begin{abstract}
In this article, a new q-generalization of the Apostol-Euler polynomials is introduced using the usual q-exponential function. We make use of such a generalization to derive several properties arising from the q-umbral calculus. (C)2017 All rights reserved.

Keywords: q-Apostol-Euler polynomials, q-numbers, q-exponential function, q-umbral calculus, $(\lambda, q)$-Euler numbers, $(\lambda, q)$-Euler polynomials, properties and identities.

2010 MSC: 11B68, 11S80, 11B65, 33D15.
\end{abstract}

\section{Introduction}

Let $\mathrm{q} \in(0,1)$. In the theory of $\mathrm{q}$-calculus, a $\mathrm{q}$-number $[\mathrm{x}]_{\mathrm{q}}$ is defined by

$$
[x]_{\mathrm{q}}=\frac{1-\mathrm{q}^{\mathrm{x}}}{1-\mathrm{q}} \text {. }
$$

Similarly, for $z \in \mathbb{C}$ with $|z|<1$, the q-exponential function $e_{\mathrm{q}}(z)$ is defined by

$$
e_{\mathrm{q}}(z)=\sum_{n=0}^{\infty} \frac{z^{n}}{[n]_{\mathrm{q}} !}
$$

In this paper, we use the following notations:

$$
[n]_{\mathrm{q}} !=[n]_{\mathrm{q}}[n-1]_{\mathrm{q}} \cdots[2]_{\mathrm{q}}[1]_{\mathrm{q}}, \quad \text { and } \quad\left(\begin{array}{l}
n \\
k
\end{array}\right)_{\mathrm{q}}=\frac{[n]_{\mathrm{q}} !}{[k]_{\mathrm{q}} ![n-k]_{\mathrm{q}} !} \text {. }
$$

\footnotetext{
*Corresponding author

Email addresses: mtsrkn@hotmail.com (Serkan Araci), acikgoz@gantep.edu.tr (Mehmet Acikgoz), tokadiag@gmail.com (Toka Diagana), harimsri@math.uvic.ca (H. M. Srivastava)

doi:10.22436/jnsa.010.04.03
}

Received 2016-09-30 
The q-integral of a function $f$ is defined by

$$
\int_{0}^{x} f(\xi) d_{q} \xi=x(1-q) \sum_{\xi=0}^{\infty} f\left(q^{\xi} x\right) q^{\xi} .
$$

For more on this and related issues, see, e.g., [4, 10-13, 15].

The q-derivative $\mathrm{D}_{\mathrm{q}}$ is defined by

$$
D_{q} f(x)=\frac{d_{q} f(x)}{d_{q} x}=\frac{f(x)-f(q x)}{(1-q) x}, \quad \text { and } \quad \lim _{q \rightarrow 1} D_{q} f(x)=\frac{d f(x)}{d x} .
$$

For a systematic study of q-derivatives, we refer the reader to [4, 10, 12, 13, 15] and [11].

Recently, Kim [11] introduced q-Euler polynomials by means of the following generating function:

$$
\sum_{n=0}^{\infty} E_{n, q}(x) \frac{t^{n}}{[n]_{q} !}=\frac{[2]_{q}}{e_{q}(t)+1} e_{q}(x t),
$$

with $E_{n, q}(0)=E_{n, q}$ (called the n-th q-Euler numbers).

Let us now consider $\lambda$ extension of $q$-Euler polynomials (or can be called $(\lambda, q)$-Euler polynomials) given by

$$
\sum_{n=0}^{\infty} E_{n, q}(x \mid \lambda) \frac{t^{n}}{[n]_{q} !}=\frac{[2]_{q}}{\lambda e_{q}(t)+1} e_{q}(x t),
$$

where $\lambda \in \mathbb{R}^{+}$.

Letting $\mathrm{q} \rightarrow 1$ in (1.1), one obtains the Apostol-Euler polynomial. Consequently, the polynomials defined by (1.1) are a new q-generalization of Euler polynomial of Apostol type. For more information on the Apostol-type polynomials, we refer the reader to recent works such as $[6,7,16,18]$ and $[28]$.

Recent investigations on this topic include an elementary and real approach to values of the Riemann zeta function [5], Apostol-Euler polynomials arising from the umbral calculus [16], a new generalization of q-Bernoulli polynomial [17], a new q-generalization of Euler numbers and polynomials using the method of Kupershmidt [11], additional theorems for the Appell polynomials and associated classes of polynomial expansions [21], theorems on Apostol-Euler polynomials arising from Euler basis [27], a new class of q-Euler and q-Bernoulli polynomials $[19,20]$, a modified q-Euler numbers of higher order with weight [22], the zeta and the q-zeta functions and associated series and integrals [29].

Let $\mathcal{F}$ be the space of all formal power series in variable $t$ over the complex number field $\mathbb{C}$, namely

$$
\mathcal{F}=\left\{f: f(t)=\sum_{k=0}^{\infty} a_{k} \frac{t^{k}}{c_{k}}, \quad\left(a_{k} \in \mathbb{C}\right)\right\},
$$

where $c_{k}$ is an admissible sequence, i.e., $c_{n} \neq 0$ for all $n \geqslant 0$, and $\mathbb{P}=\mathbb{C}[t]$ and let $\mathbb{P}^{*}$ be the vector space of all linear functionals on $\mathbb{P}$. We also denote by $\langle\mathrm{L} \mid \mathrm{p}(x)\rangle$ the action of a linear functional $L$ on the polynomial $p(x)$, which obviously satisfies the following properties:

$$
\langle\mathrm{L}+\mathrm{M} \mid \mathrm{p}(\mathrm{x})\rangle=\langle\mathrm{L} \mid \mathrm{p}(\mathrm{x})\rangle+\langle\mathrm{M} \mid \mathrm{p}(\mathrm{x})\rangle,
$$

and

$$
\langle\beta \mathrm{L} \mid \mathrm{p}(\mathrm{x})\rangle=\beta\langle\mathrm{L} \mid \mathrm{p}(\mathrm{x})\rangle,
$$

where $\beta$ is a complex constant (see, for details, $[1,9-16,26]$ and [25]).

Roman [23, 26] defined linear functionals and operators as follows:

$$
\left\langle t^{k} \mid x^{n}\right\rangle=c_{n} \delta_{n, k}, \quad(n, k \geqq 0),
$$




$$
\left\langle f(t) \mid x^{n}\right\rangle=a_{n}, \quad(n \geqq 0),
$$

and

$$
\mathrm{t}^{\mathrm{k}} \mathrm{x}^{\mathrm{n}}=\left\{\begin{array}{cc}
\frac{\mathrm{c}_{n}}{\mathrm{c}_{\mathrm{n}-\mathrm{k}}} x^{\mathrm{n}-\mathrm{k}} & \mathrm{k} \leqslant \mathrm{n} \\
0 & \mathrm{n}<\mathrm{k}
\end{array}\right.
$$

Roman also defined the following equivalent conditions in which $s_{\mathfrak{n}}(x)$ is known as Sheffer for $(g(t), t)$, where $g(t)$ is an invertible formal power series:

(S1) $\left\langle g(t) t^{k} \mid s_{n}(x)\right\rangle=c_{n} \delta_{n, k} ;$

(S2) $\mathrm{ts}_{\mathrm{n}}(\mathrm{x})=\frac{\mathrm{c}_{n}}{\mathrm{c}_{\mathrm{n}-1}} s_{\mathrm{n}-1}(\mathrm{x})$;

(S3) $\sum_{k=0}^{\infty} s_{k}(x) \frac{t^{k}}{c_{k}}=\frac{\xi(x t)}{g(t)}$, where $\xi(t)=\sum_{k=0}^{\infty} \frac{t^{k}}{c_{k}}$.

Recently, Kim and Kim $[12,13]$ considered q-umbral calculus and derived some new interesting identities for q-Bernoulli and q-Euler polynomials. The following tools about q-umbral calculus are taken from Kim and Kim's works [12, 13].

Let

$$
f_{L}(t)=\sum_{k=0}^{\infty}\left\langle L \mid x^{k}\right\rangle \frac{t^{k}}{[k]_{q} !} .
$$

Then, (1.2) gives us $\left\langle f_{L}(t) \mid x^{n}\right\rangle=\left\langle L \mid x^{n}\right\rangle$, that is, $f_{L}(t)=L$. Moreover, the map $L \longmapsto f_{L}(t)$ is an isomorphism from $\mathbb{P}^{*}$ onto $\mathcal{F}$. Henceforth $\mathcal{F}$ will denote both the algebra of formal power series in $t$ and the vector space of all linear functionals on $\mathbb{P}$. Thus an element $f(t)$ of $\mathcal{F}$ will be seen as a formal power series and a linear functional. Kim and Kim $[12,13]$ called it q-umbral algebra which is the study of the q-umbral calculus. From (1.3), we notice that

$$
\left\langle e_{q}(y t) \mid x^{n}\right\rangle=y^{n},
$$

and so

$$
\left\langle e_{\mathrm{q}}(y \mathrm{t}) \mid \mathrm{p}(\mathrm{x})\right\rangle=\mathrm{p}(\mathrm{y}), \quad(\mathrm{p}(\mathrm{x}) \in \mathbb{P}) .
$$

The order $o(f(t))$ of the power series $f(t) \neq 0$ is the smallest integer for which $a_{k}$ does not vanish. If $o(f(t))=0$, then $f(t)$ is called an invertible series. If o $(f(t))=1$, then $f(t)$ is called a delta series (see $[1-4,8-16,23-26])$. For $f(t), g(t) \in \mathcal{F}$, we have

$$
\langle f(t) g(t) \mid p(x)\rangle=\langle f(t) \mid g(t) p(x)\rangle=\langle g(t) \mid f(t) p(x)\rangle .
$$

Let $f(t) \in \mathcal{F}$ and $p(x) \in \mathbb{P}$. Then we have (see, e.g., [10-15, 26] and [25]).

$$
f(t)=\sum_{k=0}^{\infty}\left\langle f(t) \mid x^{k}\right\rangle \frac{t^{k}}{[k]_{q} !^{\prime}}, \quad \text { and } \quad p(x)=\sum_{k=0}^{\infty}\left\langle t^{k} \mid p(x)\right\rangle \frac{x^{k}}{[k]_{q} !} .
$$

Using (1.4), we obtain

$$
p^{(k)}(x)=D_{q}^{k} p(x)=\sum_{l=k}^{\infty} \frac{\left\langle t^{l} \mid p(x)\right\rangle}{[l]_{q} !} x^{l-k} \prod_{s=1}^{k}[l-s+1]_{q}
$$

which in turn yields

$$
p^{(k)}(0)=\left\langle t^{k} \mid p(x)\right\rangle, \quad \text { and } \quad\left\langle 1 \mid p^{(k)}(x)\right\rangle=p^{(k)}(0) .
$$

Thus from (1.5), we note that

$$
t^{k} p(x)=p^{(k)}(x)=D_{q}^{k} p(x)
$$


Let $f(t), g(t) \in \mathcal{F}$ with $o(f(t))=1$ and $o(g(t))=0$. Then there exists a unique sequence $s_{n}(x)$ (deg $\left.s_{n}(x)=n\right)$ of polynomials such that

$$
\left\langle g(t) f(t)^{k} \mid s_{n}(x)\right\rangle=[n]_{q} ! \delta_{n, k}, \quad(n, k \geqq 0) .
$$

The sequence $s_{\mathfrak{n}}(x)$ is called the q-Sheffer sequence for $(g(t), f(t))$, which is denoted by $s_{\mathfrak{n}}(x) \sim(g(t), f(t))$. Let $s_{n}(x) \sim(g(t), f(t))$. For $h(t) \in \mathcal{F}$ and $p(x) \in \mathbb{P}$, we have

$$
h(t)=\sum_{k=0}^{\infty} \frac{\left\langle h(t) \mid s_{k}(x)\right\rangle}{[k]_{q} !} g(t) f(t)^{k}, \quad \text { and } \quad p(x)=\sum_{k=0}^{\infty} \frac{\left\langle g(t) f(t)^{k} \mid p(x)\right\rangle}{[k]_{q} !} s_{k}(x) .
$$

The sequence $s_{n}(x)$ is Appell for $g(t)$ that

$$
s_{\mathfrak{n}}(x)=\frac{1}{g(t)} x^{n}, \quad \text { if and only if, } \quad t s_{n}(x)=[n]_{q} s_{n-1}(x), \quad \text { (see, e.g., [25]). }
$$

Furthermore, for all $y \in \mathbb{C}$, we get

$$
\frac{1}{g(\bar{f}(t)} e_{q}(y \bar{f}(t))=\sum_{k=0}^{\infty} \frac{s_{k}(y)}{[k]_{q} !} t^{k}
$$

where $\bar{f}(t)$ is the compositional inverse of $f(t)$. For further information about q-umbral calculus, we refer the reader to see Kim and Kim's works $[12,13]$.

Kim et al. (see [11-13, 15]) derived some interesting properties of the new family of q-Euler numbers and polynomials from the viewpoint of the theory of the q-calculus. On the other hand, by using the orthogonality type as defined in the umbral calculus, Kim et al. [16] derived explicit formulas for several well-known polynomials as a linear combination of the Apostol-Euler polynomials.

The main objective of this paper consists of introducing $(\lambda, q)$-Euler polynomials that seem to be a new q-generalization of Apostol-Euler polynomials. We derive several interesting properties and identities arising from the q-umbral calculus. These numbers and polynomials have interesting properties in the areas of both number theory and mathematical physics.

\section{The $(\lambda, q)$-Euler Numbers and the $(\lambda, q)$-Euler Polynomials}

Let us introduce the $(\lambda, q)$-extension of the Euler polynomials by means of the following generating function:

$$
\frac{[2]_{\mathrm{q}}}{\lambda e_{\mathrm{q}}(\mathrm{t})+1} e_{\mathrm{q}}(\mathrm{xt})=\sum_{\mathrm{n}=0}^{\infty} E_{\mathrm{n}, \mathrm{q}}(x \mid \lambda) \frac{\mathrm{t}^{\mathrm{n}}}{[\mathrm{n}]_{\mathrm{q}} !} .
$$

Clearly, if $x=0$, then $E_{n, q}(0 \mid \lambda)=E_{n, q}(\lambda)$, which are known as the $n$th $(\lambda, q)$-Euler numbers. Using (2.1), we obtain the following,

$$
E_{n, q}(x \mid \lambda)=\sum_{l=0}^{n}\left(\begin{array}{l}
n \\
l
\end{array}\right)_{q} E_{l, q}(\lambda) x^{n-l}
$$

Using (2.2), the $(\lambda, q)$-Euler numbers can be found by means of the following identities:

$$
E_{0, q}(\lambda)=\frac{2}{\lambda+1}, \quad \text { and } \quad \lambda E_{n, q}(1 \mid \lambda)+E_{n, q}(\lambda)=[2]_{q} \delta_{0, n}
$$

We immediately derive the following consequences based on (S1)-(S2) for q-polynomial with a parameter $\lambda$ defined in (2.1),

$$
E_{n, q}(x \mid \lambda) \sim\left(\frac{\lambda e_{q}(t)+1}{[2]_{q}}, t\right)
$$




$$
\mathrm{tE}_{\mathrm{n}, \mathrm{q}}(x \mid \lambda)=[\mathrm{n}]_{\mathrm{q}} \mathrm{E}_{\mathrm{n}-1, \mathrm{q}}(x \mid \lambda) .
$$

It follows from (2.3) that $E_{n, q}(x \mid \lambda)$ is Appell for $\frac{\lambda e_{q}(t)+1}{[2]_{q}}$. So, by the (1.6), we have

$$
\frac{[2]_{q}}{\lambda e_{q}(t)+1} x^{n}=E_{n, q}(x \mid \lambda) \quad(n \geqq 0) .
$$

Using (2.2), we obtain

$$
\begin{aligned}
\int_{x}^{x+y} E_{n, q}(u \mid \lambda) d_{q} u & =\sum_{l=0}^{n}\left(\begin{array}{l}
n \\
l
\end{array}\right)_{q} E_{n-l, q}(\lambda) \frac{1}{[l+1]_{q}}\left\{(x+y)^{l+1}-x^{l+1}\right\} \\
& =\frac{1}{[n+1]_{q}} \sum_{l=0}^{n}\left(\begin{array}{c}
n+1 \\
l+1
\end{array}\right)_{q} E_{n-l, q}(\lambda)\left\{(x+y)^{l+1}-x^{l+1}\right\} \\
& =\frac{1}{[n+1]_{q}}\left(E_{n+1, q}(x+y \mid \lambda)-E_{n+1, q}(x \mid \lambda)\right) .
\end{aligned}
$$

Thus, by applying (2.4), we get

$$
\begin{aligned}
\left\langle\frac{e_{\mathrm{q}}(t)-1}{t} \mid E_{n, q}(x \mid \lambda)\right\rangle & =\frac{1}{[n+1]_{q}}\left\langle\frac{e_{q}(t)-1}{t} \mid t E_{n+1, q}(x \mid \lambda)\right\rangle \\
& =\frac{1}{[n+1]_{q}}\left\{E_{n+1, q}(1 \mid \lambda)-E_{n+1, q}(\lambda)\right\} \\
& =\int_{0}^{1} E_{n, q}(u \mid \lambda) d_{q} u .
\end{aligned}
$$

Therefore, by (2.5), we obtain the following result.

Theorem 2.1. Let $\mathrm{n} \geqq 0$. Then

$$
\left\langle\frac{e_{q}(t)-1}{t} \mid E_{n, q}(x \mid \lambda)\right\rangle=\int_{0}^{1} E_{n, q}(u \mid \lambda) d_{q} u
$$

Now also, by using (1.2), we have

$$
\begin{aligned}
\left\langle\frac{\lambda e_{\mathrm{q}}(\mathrm{t})+1}{[2]_{\mathrm{q}}} \mathrm{t}^{\mathrm{k}} \mid \mathrm{E}_{\mathrm{n}, \mathrm{q}}(\mathrm{x} \mid \lambda)\right\rangle & =\frac{[\mathrm{k}]_{\mathrm{q}} !}{[2]_{\mathrm{q}}}\left(\begin{array}{l}
\mathrm{n} \\
k
\end{array}\right)_{\mathrm{q}}\left\langle\lambda e_{\mathrm{q}}(\mathrm{t})+1 \mid \mathrm{E}_{\mathrm{n}-\mathrm{k}, \mathrm{q}}(\mathrm{x} \mid \lambda)\right\rangle \\
& =[\mathrm{n}]_{\mathrm{q}} ! \delta_{\mathrm{k}, \mathrm{n}} .
\end{aligned}
$$

From the last identity, we have

$$
\left\langle\left(\frac{\lambda e_{q}(t)+1}{[2]_{q}}\right) t^{k} \mid E_{n, q}(x \mid \lambda)\right\rangle=[n]_{q} ! \delta_{n, k} .
$$

Let

$$
\mathbb{P}_{n}=\{q(x) \in \mathbb{C}[x] \mid \operatorname{deg} q(x) \leqslant n\} .
$$

Also, for $\mathrm{q}(\mathrm{x}) \in \mathbb{P}_{n}$, we assume that

$$
q(x)=\sum_{k=0}^{n} b_{k, q} E_{k, q}(x \mid \lambda) .
$$


It follows from (2.6) and (2.7) that

$$
\begin{aligned}
\left\langle\frac{\lambda e_{\mathrm{q}}(\mathrm{t})+1}{[2]_{\mathrm{q}}} \mathrm{t}^{\mathrm{k}} \mid \mathrm{q}(\mathrm{x})\right\rangle & =\sum_{\mathrm{l}=0}^{n} b_{\mathrm{l}, \mathrm{q}}\left\langle\frac{\lambda e_{\mathrm{q}}(\mathrm{t})+1}{[2]_{\mathrm{q}}} \mathrm{t}^{\mathrm{k}} \mid \mathrm{E}_{\mathrm{l}, \mathrm{q}}(\mathrm{x} \mid \lambda)\right\rangle \\
& =\sum_{\mathrm{l}=0}^{n} b_{\mathrm{l}, \mathrm{q}}[\mathrm{l}]_{\mathrm{q}} ! \delta_{\mathrm{l}, \mathrm{k}}=[\mathrm{k}]_{\mathrm{q}} ! \mathrm{b}_{\mathrm{k}, \mathrm{q}} .
\end{aligned}
$$

Furthermore

$$
b_{k, q}=\frac{1}{[k]_{q} !}\left\langle\frac{\lambda e_{q}(t)+1}{[2]_{q}} t^{k} \mid q(x)\right\rangle=\frac{1}{[2]_{q}[k]_{q} !}\left\{\lambda q^{(k)}(1)+q^{(k)}(0)\right\},
$$

where

$$
q^{(k)}(x)=D_{q}^{k} q(x) .
$$

Therefore, by (2.7) and (2.8), we obtain the following theorem.

Theorem 2.2. For $\mathrm{q}(\mathrm{x}) \in \mathbb{P}_{n}$, let

$$
q(x)=\sum_{k=0}^{n} b_{k, q} E_{k, q}(x \mid \lambda)
$$

Then

$$
b_{k, q}=\frac{1}{[2]_{q}[k]_{q} !}\left\{\lambda q^{(k)}(1)+q^{(k)}(0)\right\} .
$$

The q-Bernoulli polynomials $B_{n, q}(x)$ are defined by

$$
\frac{t}{e_{q}(t)-1} e_{q}(x t)=\sum_{n=0}^{\infty} B_{n, q}(x) \frac{t^{n}}{[n]_{q} !}
$$

From this, we have for $n \geqq 0$,

$$
\begin{aligned}
B_{n, q}(x) & \sim\left(\frac{e_{q}(t)-1}{t}, t\right) \\
t B_{n, q}(x) & =[n]_{q} B_{n-1, q}(x), \quad \text { (see [12] for details). }
\end{aligned}
$$

Let us take

$$
\mathrm{q}(x)=\mathrm{B}_{\mathrm{n}, \mathrm{q}}(\mathrm{x}) \in \mathbb{P}_{n} .
$$

Then $B_{n, q}(x)$ can be generated as a linear combination of

$$
\left\{E_{0, q}(x \mid \lambda), E_{1, q}(x \mid \lambda), \cdots, E_{n, q}(x \mid \lambda)\right\},
$$

as follows:

$$
B_{n, q}(x)=\sum_{k=0}^{n} b_{k, q} E_{k, q}(x \mid \lambda)
$$

where

$$
\begin{aligned}
b_{k, q} & =\frac{1}{[2]_{q}[k]_{q} !}\left\langle\left(\lambda e_{q}(t)+1\right) t^{k} \mid B_{n, q}(x)\right\rangle \\
& =\frac{[n]_{q}[n-1]_{q} \cdots[n-k+1]_{q}}{[2]_{q}[k]_{q} !}\left\langle\lambda e_{q}(t)+1 \mid B_{n-k, q}(x)\right\rangle
\end{aligned}
$$




$$
\begin{aligned}
& =\frac{1}{[2]_{\mathrm{q}}}\left(\begin{array}{l}
n \\
k
\end{array}\right)_{\mathrm{q}}\left\langle\lambda e_{\mathrm{q}}(\mathrm{t})+1 \mid \mathrm{B}_{n-k, \mathrm{q}}(x)\right\rangle \\
& =\frac{1}{[2]_{\mathrm{q}}}\left(\begin{array}{l}
n \\
k
\end{array}\right)_{\mathrm{q}}\left(\lambda \mathrm{B}_{n-k, \mathrm{q}}(1)+\mathrm{B}_{n-k, \mathrm{q}}\right),
\end{aligned}
$$

where $B_{n, q}:=B_{n, q}(0)$ are called q-Bernoulli numbers, e.g., see [12]. Kim et al. [13] derived the following identity:

$$
\mathrm{B}_{0, \mathrm{q}}=1, \quad \text { and } \quad \mathrm{B}_{\mathrm{n}, \mathrm{q}}(1)-\mathrm{B}_{\mathrm{n}, \mathrm{q}}=\left\{\begin{array}{lc}
1 & (\mathrm{n}=1), \\
0 & (\mathrm{n}>1)
\end{array}\right.
$$

Since $B_{1, q}=-\frac{1}{[2]_{q}}$, by (2.9) and (2.10), we have

$$
\begin{aligned}
B_{n, q}(x) & =b_{n, q} E_{n, q}(x \mid \lambda)+b_{n-1} E_{n-1, q}(x \mid \lambda)+\sum_{k=0}^{n-2} b_{k, q} E_{k, q}(x \mid \lambda) \\
& =\frac{\lambda+1}{[2]_{q}} E_{n, q}(x \mid \lambda)+\frac{[n]_{q}}{[2]_{q}^{2}}(\lambda q-1) E_{n-1, q}(x \mid \lambda)+\frac{\lambda+1}{[2]_{q}} \sum_{k=0}^{n-2}\left(\begin{array}{l}
n \\
k
\end{array}\right)_{q} B_{n-k, q} E_{k, q}(x \mid \lambda) .
\end{aligned}
$$

The previous facts can be formulated as follows:

Theorem 2.3. Let $\mathrm{n} \geqq 2$. Then

$$
\begin{aligned}
B_{n, q}(x)= & \frac{\lambda+1}{[2]_{q}} E_{n, q}(x \mid \lambda)+\frac{[n]_{q}}{[2]_{q}^{2}}(\lambda q-1) E_{n-1, q}(x \mid \lambda) \\
& +\frac{\lambda+1}{[2]_{q}} \sum_{k=0}^{n-2}\left(\begin{array}{l}
n \\
k
\end{array}\right)_{q} B_{n-k, q} E_{k, q}(x \mid \lambda) .
\end{aligned}
$$

For $r \in \mathbb{Z}_{\geqq 0}$, the higher-order $(\lambda, q)$-Euler polynomials $E_{n, q}^{(r)}(x \mid \lambda)$ are defined by the following $q$ Taylor expansion at $\mathrm{t}=0$ :

$$
\left(\frac{[2]_{q}}{\lambda e_{q}(t)+1}\right)^{r} e_{q}(x t)=\sum_{n=0}^{\infty} E_{n, q}^{(r)}(x \mid \lambda) \frac{t^{n}}{[n]_{q} !} .
$$

In the case, $x=0, E_{n, q}^{(r)}(0 \mid \lambda)=E_{n, q}^{(r)}(\lambda)$ are called the $r$ th higher-order $(\lambda, q)$-Euler numbers.

Let

$$
g^{r}(t \mid \lambda)=\left(\frac{\lambda e_{q}(t)+1}{[2]_{q}}\right)^{r} .
$$

It is clear that $g^{r}(t \mid \lambda)$ is an invertible series. It follows from (2.11) that $E_{n, q}(x \mid \lambda)$ is Appell for $\left(\frac{\lambda e_{q}(t)+1}{[2]_{q}}\right)^{r}$. So, by (1.6), we have

$$
E_{n, q}^{(r)}(x \mid \lambda)=\frac{1}{g^{r}(t \mid \lambda)} x^{n}
$$

and

$$
t E_{n, q}^{(r)}(x \mid \lambda)=[n]_{q} E_{n-1, q}^{(r)}(x \mid \lambda)
$$

Thus, we have

$$
E_{n, q}^{(r)}(x \mid \lambda) \sim\left(\left(\frac{\lambda e_{q}(t)+1}{[2]_{q}}\right)^{r}, t\right) .
$$


By (1.2) and (2.11), we get

$$
\left\langle\frac{[2]_{q}^{r}}{\left(\lambda e_{q}(t)+1\right)^{r}} e_{q}(y t) \mid x^{n}\right\rangle=E_{n, q}^{(r)}(y \mid \lambda)=\sum_{l=0}^{n}\left(\begin{array}{c}
n \\
l
\end{array}\right)_{q} E_{n-l, q}^{(r)}(\lambda) y^{l} .
$$

We thus find that

$$
\begin{aligned}
\left\langle\left(\frac{[2]_{\mathrm{q}}}{\lambda e_{\mathrm{q}}(\mathrm{t})+1}\right)^{\mathrm{r}} \mid x^{\mathrm{n}}\right\rangle & =\left\langle\frac{[2]_{\mathrm{q}}}{\lambda e_{\mathrm{q}}(\mathrm{t})+1} \cdots \frac{[2]_{\mathrm{q}}}{\lambda e_{\mathrm{q}}(\mathrm{t})+1} \mid x^{n}\right\rangle \\
& =\sum_{i_{1}+\cdots+i_{r}=n}\left(\begin{array}{c}
n \\
i_{1}, \cdots, i_{r}
\end{array}\right)_{\mathrm{q}} \mathrm{E}_{i_{1}, \mathrm{q}}(\lambda) \cdots \mathrm{E}_{\mathrm{i}_{\mathrm{r}}, \mathrm{q}}(\lambda),
\end{aligned}
$$

where

By using (2.12), we have

$$
\left(\begin{array}{c}
n \\
i_{1}, \cdots, i_{r}
\end{array}\right)_{q}=\frac{[n]_{q} !}{\left[i_{1}\right]_{q} ! \cdots\left[i_{r}\right]_{q} !}
$$

$$
\left\langle\left(\frac{[2]_{\mathrm{q}}}{\lambda e_{\mathrm{q}}(\mathrm{t})+1}\right)^{\mathrm{r}} \mid x^{\mathfrak{n}}\right\rangle=\mathrm{E}_{\mathrm{n}, \mathrm{q}}^{(\mathrm{r})}(\lambda) .
$$

Therefore, by (2.13) and (2.14), we obtain the following theorem.

Theorem 2.4. Let $\mathrm{n} \geqq 0$. Then

$$
E_{n, q}^{(r)}(\lambda)=\sum_{i_{1}+\cdots+i_{r}=n}\left(\begin{array}{c}
n \\
i_{1}, \cdots, i_{r}
\end{array}\right)_{q} E_{i_{1}, q}(\lambda) \cdots E_{i_{r}, q}(\lambda) .
$$

Let us take

$$
q(x)=E_{n, q}^{(r)}(x \mid \lambda) \in \mathbb{P}_{n}
$$

Then, by Theorem 2.2, we write

$$
E_{n, q}^{(r)}(x \mid \lambda)=\sum_{k=0}^{n} b_{k, q} E_{k, q}(x \mid \lambda),
$$

where the coefficient $b_{k, q}$ is given by

$$
\begin{aligned}
b_{k, q} & =\frac{1}{[2]_{q}[k]_{q} !}\left\langle\left(\lambda e_{q}(t)+1\right) t^{k} \mid q(x)\right\rangle=\frac{1}{[2]_{q}[k]_{q} !}\left\langle\left(\lambda e_{q}(t)+1\right) t^{k} \mid q(x)\right\rangle \\
& =\frac{\left(\begin{array}{l}
n \\
k
\end{array}\right)_{q}}{[2]_{q}}\left\langle\left(\lambda e_{q}(t)+1\right) \mid E_{n-k, q}^{(r)}(x \mid \lambda)\right\rangle=\frac{\left(\begin{array}{l}
n \\
k
\end{array}\right)_{q}}{[2]_{q}}\left(\lambda E_{n-k, q}^{(r)}(1 \mid \lambda)+E_{n-k, q}^{(r)}(\lambda)\right) .
\end{aligned}
$$

From (2.11), we have

$$
\begin{aligned}
\sum_{k=0}^{\infty}\left(\lambda E_{n, q}^{(r)}(1 \mid \lambda)+E_{n, q}^{(r)}(\lambda)\right) \frac{t^{n}}{[n]_{q} !} & =\left(\frac{[2]_{q}}{\lambda e_{q}(t)+1}\right)^{r}\left(\lambda e_{q}(t)+1\right) \\
& =[2]_{q}\left(\frac{[2]_{q}}{\lambda e_{q}(t)+1}\right)^{r-1} \\
& =\sum_{n=0}^{\infty}[2]_{q} E_{n, q}^{(r-1)}(\lambda) \frac{t^{n}}{[n]_{q} !}
\end{aligned}
$$

By comparing the coefficients $\frac{t^{n}}{n !}$ in the above equation, we get

$$
\lambda E_{n, q}^{(r)}(1 \mid \lambda)+E_{n, q}^{(r)}(\lambda)=[2]_{q} E_{n, q}^{(r-1)}(\lambda) .
$$

Thus, clearly, the following theorem is derivable by applying (2.15) and (2.16). 
Theorem 2.5. Let $\mathrm{n} \in \mathrm{Z}_{\geqq 0}$ and $\mathrm{r} \in \mathrm{Z}_{>0}$. Then

$$
E_{n, q}^{(r)}(x \mid \lambda)=\sum_{k=0}^{n}\left(\begin{array}{l}
n \\
k
\end{array}\right)_{q} E_{n-k, q}^{(r-1)}(\lambda) E_{k, q}(x \mid \lambda) .
$$

Let us assume that

$$
q(x)=\sum_{k=0}^{n} b_{k, q}^{r} E_{k, q}^{(r)}(x \mid \lambda) \in \mathbb{P}_{n} .
$$

By a similar method, we find the coefficient $b_{k, q}^{r}$ as follows:

$$
b_{k, q}^{r}=\frac{1}{[2]_{q}^{r}[k]_{q} !} \sum_{l=0}^{r}\left(\begin{array}{l}
r \\
l
\end{array}\right) \lambda^{l} \sum_{m \geqq 0} \sum_{i_{1}+\cdots+i_{l}=m}\left(\begin{array}{c}
m \\
i_{1}, \cdots, i_{l}
\end{array}\right)_{q} \frac{1}{[m]_{q} !} q^{(m+k)}(0) .
$$

Therefore, we obtain the following theorem.

Theorem 2.6. For $\mathrm{n} \geqq 0$, let

$$
q(x)=\sum_{k=0}^{n} b_{k, q}^{r} E_{k, q}^{(r)}(x \mid \lambda) \in P_{n}
$$

Then

$$
\begin{aligned}
b_{k, q}^{r} & =\frac{1}{[2]_{q}^{r}[k]_{q} !}\left\langle\left(\lambda e_{q}(t)+1\right)^{r} t^{k} \mid q(x)\right\rangle \\
& =\frac{1}{[2]_{q}^{r}[k]_{q} !} \sum_{m \geqq 0} \sum_{l=0}^{r}\left(\begin{array}{l}
r \\
l
\end{array}\right) \lambda^{l} \sum_{i_{1}+\cdots+i_{l}=m}\left(\begin{array}{c}
m \\
i_{1}, \cdots, i_{l}
\end{array}\right)_{q} \frac{1}{[m]_{q} !} q^{(m+k)}(0),
\end{aligned}
$$

where

$$
q^{(k)}(x)=D_{q}^{k} q(x) .
$$

Let us consider $q(x)=E_{n, q}(x \mid \lambda) \in \mathbb{P}_{n}$. Then, by Theorem 2.6, we write

$$
E_{n, q}(x \mid \lambda)=\sum_{k=0}^{n} b_{k, q}^{r} E_{k, q}^{(r)}(x \mid \lambda) \text {. }
$$

By applying Theorem 2.6 and (2.17), we are led to the following result.

Theorem 2.7. For $\mathrm{n}, \mathrm{r} \geqq 0$, the following assertion holds true:

$$
\begin{aligned}
E_{n, q}(x \mid \lambda)= & \frac{1}{[2]_{q}^{r}} \sum_{k=0}^{n}\left(\sum_{m=0}^{n-k} \sum_{l=0}^{r} \sum_{i_{1}+\cdots+i_{l}=m} \lambda^{l}\left(\begin{array}{l}
r \\
l
\end{array}\right)\left(\begin{array}{c}
m \\
i_{1}, \cdots, i_{l}
\end{array}\right)_{q}\left(\begin{array}{c}
m+k \\
m
\end{array}\right)_{q}\left(\begin{array}{c}
n \\
m+k
\end{array}\right)_{q}\right. \\
& \left.\cdot E_{n-m-k, q}(\lambda)\right) E_{k, q}^{(r)}(x \mid \lambda) .
\end{aligned}
$$

\section{Concluding remarks and observations}

We have investigated various properties of a new q-generalization of the Apostol-Euler polynomials which we introduced by using the usual q-exponential function $e_{\mathrm{q}}(\mathrm{x})$. These properties and other related identities are shown to arise from the q-umbral calculus. Two of the main results presented in our investigation (Theorem 2.2 and Theorem 2.6) seem to be sufficiently deep and general for obtaining not only new, but also interesting, identities related to some special polynomials in terms of other new qgeneralizations of the Euler polynomials of the Apostol type. 


\section{References}

[1] S. Araci, Novel identities involving Genocchi numbers and polynomials arising from applications of umbral calculus, Appl. Math. Comput., 233 (2014), 599-607. 1, 1

[2] S. Araci, M. Acikgoz, A. Kilicman, Extended p-adic q-invariant integrals on $\mathbb{Z}_{\mathrm{p}}$ associated with applications of umbral calculus, Adv. Difference Equ., 2013 (2013), 14 pages.

[3] S. Araci, M. Acikgoz, E. Şen, On the extended Kim's p-adic q-deformed fermionic integrals in the p-adic integer ring, J. Number Theory, 133 (2013), 3348-3361.

[4] S. Araci, M. Acikgoz, J. J. Seo, A new family of q-analogue of Genocchi numbers and polynomials of higher order, Kyungpook Math. J., 54 (2014), 131-141. 1, 1

[5] A. G. Bagdasaryan, An elementary and real approach to values of the Riemann zeta function, Phys. Atom. Nucl., 73 (2010), 251-254. 1

[6] J.-S. Choi, P. J. Anderson, H. M. Srivastava, Some q-extensions of the Apostol-Bernoulli and the Apostol-Euler polynomials of order $\mathrm{n}$, and the multiple Hurwitz zeta function, Appl. Math. Comput., 199 (2008), 723-737. 1

[7] J.-S. Choi, P. J. Anderson, H. M. Srivastava, Carlitz's q-Bernoulli and q-Euler numbers and polynomials and a class of generalized q-Hurwitz zeta functions, Appl. Math. Comput., 215 (2009), 1185-1208. 1

[8] R. Dere, Y. Simsek, H. M. Srivastava, A unified presentation of three families of generalized Apostol type polynomials based upon the theory of the umbral calculus and the umbral algebra, J. Number Theory, 133 (2013), 3245-3263. 1

[9] E. C. Ihrig, M. E. H. Ismail, A q-umbral calculus, J. Math. Anal. Appl., 84 (1981), 178-207. 1

[10] M. E. H. Ismail, M. Rahman, Inverse operators, q-fractional integrals, and q-Bernoulli polynomials, J. Approx. Theory, 114 (2002), 269-307. 1, 1

[11] T. Kim, q-generalized Euler numbers and polynomials, Russ. J. Math. Phys., 13 (2006), 293-298. 1, 1, 1

[12] D. S. Kim, T. Kim, q-Bernoulli polynomials and q-umbral calculus, Sci. China Math., 57 (2014), 1867-1874. 1, 1, 1, 2, 2

[13] D. S. Kim, T. Kim, Some identities of q-Euler polynomials arising from q-umbral calculus, J. Inequal. Appl., 2014 (2014), 12 pages. $1,1,1,2$

[14] D. S. Kim, T. Kim, Umbral calculus associated with Bernoulli polynomials, J. Number Theory, 147 (2015), 871-882.

[15] D. S. Kim, T. Kim, S.-H. Lee, J.-J. Seo, A note on q-Frobenius-Euler numbers and polynomials, Adv. Studies Theor. Phys., 7 (2013), 881-889. 1, 1, 1

[16] T. Kim, T. Mansour, S.-H. Rim, S.-H. Lee, Apostol-Euler polynomials arising from umbral calculus, Adv. Difference Equ., 2013 (2013), 7 pages. 1, 1, 1

[17] B. A. Kupershmidt, Reflection symmetries of q-Bernoulli polynomials, J. Nonlinear Math. Phys., 12 (2005), 412-422. 1

[18] Q.-M. Luo, The multiplication formulas for the Apostol-Bernoulli and Apostol-Euler polynomials of higher order, Integral Transforms Spec. Funct., 20 (2008), 377-391. 1

[19] N. I. Mahmudov, On a class of q-Bernoulli and q-Euler polynomials, Adv. Difference Equ., 2013 (2013), 11 pages. 1

[20] N. I. Mahmudov, M. E. Keleshteri, On a class of generalized q-Bernoulli and q-Euler polynomials, Adv. Difference Equ., 2013 (2013), 10 pages. 1

[21] Á. Pintér, H. M. Srivastava, Addition theorems for the Appell polynomials and the associated classes of polynomial expansions, Aequationes Math., 85 (2013), 483-495. 1

[22] S.-H. Rim, J.-H. Jeong, On the modified q-Euler numbers of higher order with weight, Adv. Stud. Contemp. Math. (Kyungshang), 22 (2012), 93-98. 1

[23] S. Roman, The theory of the umbral calculus, I, J. Math. Anal. Appl., 87 (1982), 58-115. 1, 1

[24] S. Roman, The theory of the umbral calculus, III, J. Math. Anal. Appl., 95 (1983), 528-563.

[25] S. Roman, The umbral calculus, Pure and Applied Mathematics, Academic Press, Inc. [Harcourt Brace Jovanovich, Publishers], New York, (1984). 1, 1, 1.6

[26] S. Roman, More on the umbral calculus, with emphasis on the q-umbral calculus, J. Math. Anal. Appl., 107 (1985), 222-254. 1, 1

[27] E. Şen, Theorems on Apostol-Euler polynomials of higher order arising from Euler basis, Adv. Stud. Contemp. Math. (Kyungshang), 23 (2013), 337-345. 1

[28] H. M. Srivastava, Some generalizations and basic (or q-) extensions of the Bernoulli, Euler and Genocchi polynomials, Appl. Math. Inf. Sci., 5 (2011), 390-444. 1

[29] H. M. Srivastava, J.-S. Choi, Zeta and q-Zeta functions and associated series and integrals, Elsevier, Inc., Amsterdam, (2012). 1 